\title{
Promoting functional independence in people with Alzheimer's disease: outcomes of a home-based occupational therapy intervention in Spain
}

\author{
Adriana Ávila ${ }^{1}$, Iván De-Rosende-Celeiro ${ }^{1}$, Gabriel Torres ${ }^{2}$, Mirian Vizcaíno ${ }^{1}$, Manuel \\ Peralbo $^{3}$, Montserrat Durán ${ }^{3}$ \\ ${ }^{1}$ Departamento de Ciencias da Saúde, Unidade de Investigación de Terapia Ocupacional en intervencións non \\ farmacolóxicas, Universidade da Coruña, A Coruña, Spain \\ ${ }^{2}$ Departamento de Educación Física e Deportiva, Universidade da Coruña, A Coruña, Spain \\ ${ }^{3}$ Departamento de Psicoloxía, Universidade da Coruña, A Coruña, Spain
}

\begin{abstract}
Nonpharmacologic therapies such as occupational therapy (OT) are promising for people with Alzheimer's disease (AD). However, more research is needed to better understand the effectiveness of home-based OT programs. This pilot study aimed to assess the effects of a home-based, high-intensity and multicomponent OT intervention on the activities of daily living of people with AD in Spain. The secondary objective was to examine its impact on the cognitive functions. A multiple-baseline intrasubject design was used. Twenty-one community-dwelling older adults with mild $\mathrm{AD}$ (mean age 78.6 years) and their primary caregivers participated in a 12-week home-based OT program. This intervention was replicated for 8 weeks after a 1.5 -month intervention withdrawal period. The intervention followed a holistic, biopsychosocial and client-centred approach and consisted of the following components: meaningful activities/tasks, cognitive stimulation, activation of psychomotor and sensory skills, home modification, caregiver counselling and training in daily living skills. Functional independence was the primary outcome (Barthel Index). The cognitive functions were assessed by the Loewenstein Occupational Therapy Cognitive AssessmentGeriatric (LOTCA-G). Data were analysed using nonparametric tests. Main results showed that after completing the OT program, 6.5 months after the moment of inclusion, the level of functional independence improved significantly and the effect size was large. Moreover, there was a significant moderate-to-substantial improvement in several cognitive functions after each of the two intervention periods: place orientation, time orientation and attention/concentration. In summary, the findings give a great deal of information as a basis for further research. This study provides evidence that an intensive home-based OT intervention has a positive influence on daily activities and some cognitive functions, suggesting that this program may be beneficial as a nonpharmacological supplementary tool in health and social care for people with AD living in the community.
\end{abstract}

Keywords

Activities of daily living; Alzheimer's disease; Cognition; Occupational therapy; Treatment outcome 


\section{INTRODUCTION}

Alzheimer's disease (AD) is the most common cause of dementia and a major health and social problem for older people. Its prevalence in Europe has been estimated at $5.05 \%$ in a recent meta-analysis (Niu, Álvarez-Álvarez, Guillén-Grima, \& Aguinaga-Ontoso, 2016). In the United States, an estimated 5.4 million people have AD (Alzheimer's Association, 2016) and dementia affects $8.8 \%$ of the people older than 65 years (Langa et al., 2017). Moreover, the prevalence is expected to increase drastically in the next decades (Alzheimer's Association, 2016). AD is an irreversible process that produces a substantial impact on daily functioning. People with AD experience progressive mental and motor impairments, reduced participation and long-term functional dependence. Disability in the basic activities of daily living (ADL) impacts on the quality of life of the caregivers and is a significant burden on healthcare systems (Alzheimer's Association, 2016). Impairments in ADL have been associated with higher costs of health and social care (Reed et al., 2016) and a greater number of hours of informal care (Zhu et al., 2008).

Nonpharmacologic therapies are a crucial part of AD care. A systematic review concluded that these strategies can delay progression of functional decline in people with dementia (McLaren, Lamantia, \& Callahan, 2013). Despite the lack of disease-modifying strategies, these interventions play a key role on disability in ADL and have great potential to promote quality of life, although more research is needed to better analyse their effectiveness (Alzheimer's Association, 2016). Three major groups of nonpharmacological therapies have been established: occupational therapy (OT), exercise programs and multi-faceted interventions (McLaren et al., 2013). The present study addressed the outcomes of a homebased OT intervention. OT focuses on function and its main method of intervention includes an individualised combination of therapeutically selected occupations and activities that are designed to enable or maintain the highest possible level of functioning and autonomy. The primary aim is to promote optimal levels of engagement in meaningful occupations related to health, well-being and participation (American Occupational Therapy Association, 2014). OT interventions are client-centred and use a holistic and collaborative approach based on the needs of the client-caregiver dyad (Schaber \& Lieberman, 2010).

Previous research has examined the effects of the OT programs for people with dementia. Most studies have focused on behavioural problems, emotional functions and cognitive abilities. A metaanalysis has concluded that OT based on sensory stimulation improves behavioural symptoms (Kim, Yoo, Jung, Park, \& Park, 2012). In people with moderate and severe dementia, a systematic review has revealed that OT impacts on the quality of the life and emotional problems of patients and caregivers (Korczak, Habermann, \& Braz, 2013). In day care units and residential centres, several studies have reported that OT reduces cognitive, emotional and behavioural problems (Bach, Bach, Bohmer, Fruhwald, \& Grilc, 1995; Cho et al., 2015; Lam et al., 2010; Lim et al., 2012; Ng et al., 2006; Rogers et al., 1999). Regarding home-based OT interventions, other investigations have revealed improvements in the behavioural functions of people with dementia (Gitlin, Corcoran, Winter, Boyce, \& Hauck, 2001), as well as positive outcomes on the level of burden, the sense of competence and quality of life of caregivers (Dooley \& Hinojosa, 2004; Graff et al., 2006).

Despite the progressive functional decline in ADL of people with AD, relatively little is known about the role of OT in improving and maintaining functional independence in self-care. An OT program increased active participation in ADL of severely cognitively impaired individuals in a residential setting (Rogers et al., 1999). In community centres, the literature has not found evidence that OT improves the performance of self-care in people with mild cognitive impairment (Ng et al., 2006) or dementia (Baldelli et al., 2007; Cho et al., 2015; Lam et al., 2010). Furthermore, research on the effects of home-based OT strategies on ADL among people with dementia is scarce, identifying few clinical trials with conflicting results. In a study on the effects of an OT home intervention, the subjects who received OT improved their daily instrumental activities, but there were no significant differences in ADL between the experimental and control groups (Gitlin et al., 2001). In contrast, another randomised clinical trial (RCT) found a significant improvement in ADL among people who participated in a home-based program implemented by occupational therapists (Graff et al., 2006). Recently, a RCT compared a control group receiving best-practice primary care with an intervention group receiving best-practice primary care plus 
a home-based OT intervention. At 24 months, functional independence declined in both groups and no differences in ADL between the experimental and control groups were observed (Callahan et al., 2017). Accordingly, there is a critical need to better understand the effectiveness of OT interventions on disabilities in ADL. In addition, most OT studies have not been conducted in the home environment and, therefore, the effects of home-based OT programs as an adjuvant therapy for functional and cognitive problems have been under-explored.

Based on the abovementioned findings, we have developed a home-based OT intervention for community-dwelling older people with mild AD. In this pilot study, we conducted a preliminary investigation of its effectiveness. The main objective was to assess the effects on functional independence in ADL. The secondary aim was to examine its impact on the cognitive functions. In the next section, we analyse the key characteristics of the intervention applied. We tested the hypothesis that people with AD would significantly improve the functional and cognitive abilities following participation in this program. Finally, preliminary results of this intervention are discussed and we conclude with an analysis of the study implications for practice in the community.

\section{METHODS}

\subsection{Study design}

To examine the effects of the OT intervention on functional independence and cognitive abilities, a multiple-baseline intrasubject design was used (Hersen \& Barlow, 1976; Kazdin, 1980). The intervention was replicated after a 1.5 -month intervention withdrawal period.

\subsection{Participants}

This study was conducted from 2007 to 2012 on a sample of 21 community-dwelling older adults with $\mathrm{AD}$ and their 21 primary informal caregivers. The participants were outpatients controlled by AD in public and private hospitals of three cities in the Spanish autonomous region of Galicia (Coruña, Santiago de Compostela and Lugo), in north-western Spain, and they were recruited using a convenience sampling method by neurologists and geriatricians working in these hospitals.

The eligibility criteria were: (a) community-dwelling individuals aged $>70$ years; (b) a formal diagnosis of possible or probable AD made by a neurologist or geriatrician, according to the Diagnostic and Statistical Manual of Mental Disorder, Fourth Edition (American Psychiatric Association, 2000); (c) in Stage 4 (mild dementia) of the Global Deterioration Scale (GDS) (Reisberg, Ferris, De Leon, \& Crook, 1982); this staging was carried out by the medical specialist taking into account all cognitive, functional and behavioural data obtained in a clinical interview; (d) the physical and mental condition in order to understand and follow the assessment and intervention procedures; (e) the subjects were not receiving any form of OT intervention or other interventions classified as nonpharmacological therapies in a previous systematic review (McLaren et al., 2013) (e.g. cognitive stimulation); and (f) people who were not using day care services. Subjects with cognitive comorbidity (e.g. brain injury or intellectual disability), terminal illness, significant sensory or communicative impairments (e.g. aphasia or blindness) and severe behavioural symptoms, were excluded. Primary caregivers were at least 18 years old and were willing to participate in the research. The doctors gave all eligible participants and caregivers oral and written study information.

This study was conducted as part of a larger doctoral thesis on the effectiveness of home-based OT among people with neurological diseases presented at the University of Coruña. After receiving university-based institutional review board approval, the participating subjects with AD and their caregivers provided written informed consent and were informed that they could withdraw from the study at any time. The study followed the Declaration of Helsinki (World Medical Association, 2013). 
Confidentiality was preserved in accordance with the Spanish Data Protection Law. Socio-demographic data were recorded for descriptive purposes (Table 1).

Table 1. Socio-demographic characteristics of the study population at initial assessment $(n=21)$

\begin{tabular}{lc}
\hline Sample characteristics & Value, $n(\%)$ \\
\hline & \\
Age (year) & $78.6(3.3)$ \\
Mean (SD) & $71-85$ \\
Range & \\
Gender & $14(66.6)$ \\
Women & \\
Marital status & $11(52.4)$ \\
Married & $6(28.6)$ \\
Widow/widower & $4(19.0)$ \\
Single & \\
Educational level & \\
Primary education & $7(33.3)$ \\
Secondary education & $11(52.4)$ \\
Tertiary education & $3(14.3)$ \\
Living arrangement & \\
Living alone & $1(4.7)$ \\
Living with spouse only & $11(52.4)$ \\
Living with others & $9(42.9)$ \\
Type of housing & \\
Multidwelling house & $12(57.1)$ \\
Single-family house & $9(42.9)$ \\
Living area & \\
Highly urban or semiurban & $17(81.0)$ \\
Rural & $4(19.0)$ \\
\hline
\end{tabular}

Notes. SD, standard deviation.

${ }^{a}$ United Nations Educational, Scientific, and Cultural Organization (2006).

\subsection{Outcome measures}

The Barthel Index (BI) (Mahoney \& Barthel, 1965) was the primary outcome. It is widely used to assess the ability to perform ADL without personal assistance (Table 2). These activities are scored in steps of five points on an ordinal scale ranging from dependence to independence: bathing and grooming are scored 0 or 5 ; feeding, bladder control, bowel control, toileting, dressing and stairs are scored 0,5 or 10; transfers and walking are scored $0,5,10$ or 15. Each subject was assessed on his/her ADL performance through direct observations. The items are summed and the total score ranges from 0 (total dependence) to 100 (total independence). This scale is easy to administer, quick and requires little training. The BI has been used in the ADL assessment of people with dementia previously (Baldelli et al., 2007; Cho et al., 2015; Ng et al., 2006; Rogers et al., 1999). 
Table 2. Functional independence in ADL before and after the home-based OT intervention $(n=21)$

\begin{tabular}{|c|c|c|c|c|}
\hline & \multicolumn{2}{|l|}{ Preintervention $\left(\mathrm{T}_{1}\right)$} & \multicolumn{2}{|l|}{ Postintervention $\left(\mathrm{T}_{4}\right)$} \\
\hline & Independence, $n(\%)$ & Median (Q1Q3) & Independence, $n(\%)$ & Median (Q1Q3) \\
\hline Dressing & $2(9.5)$ & $5(5-5)$ & $11(52.4)$ & $10(5-10)$ \\
\hline Bathing & $6(28.6)$ & $0(0-5)$ & $8(38.1)$ & $0(0-5)$ \\
\hline Grooming & $12(57.1)$ & $5(0-5)$ & $16(76.2)$ & $5(2.5-5)$ \\
\hline Stairs & $16(76.2)$ & $10(7.5-10)$ & $16(76.2)$ & $10(7.5-10)$ \\
\hline Toileting & $18(85.7)$ & $10(10-10)$ & $20(95.2)$ & $10(10-10)$ \\
\hline Feeding & $18(85.7)$ & $0(0-5)$ & $18(85.7)$ & $0(0-5)$ \\
\hline Bladder & $20(95.2)$ & $10(10-10)$ & $20(95.2)$ & $10(10-10)$ \\
\hline Bowels & $21(100)$ & $10(10-10)$ & $21(100)$ & $10(10-10)$ \\
\hline Walking & $21(100)$ & $15(15-15)$ & $21(100)$ & $15(15-15)$ \\
\hline Transfers & $21(100)$ & $15(15-15)$ & $21(100)$ & $15(15-15)$ \\
\hline
\end{tabular}

Notes. Values based on the Barthel Index.

Bold values denote statistical significance.

ADL: basic activities of daily living; OT: occupational therapy; $\mathrm{T}_{1}$ : before the intervention; $\mathrm{T}_{4}$ : after completion of the second intervention period; Q1: first quartile; Q3: third quartile.

The secondary outcome measure was the Loewenstein Occupational Therapy Cognitive AssessmentGeriatric (LOTCA-G) (Itzkovich, Elazar, \& Katz, 1996), a cognitive assessment tool adapted for the older population. This 24 -item test is widely used by occupational therapists and evaluates eight cognitive areas (Table 3). Each subtest is assessed with an ordinal scale from 1 to 4 , except for the orientation items, which are evaluated from 1 to 8 . A higher score means better cognitive performance. This test discriminates between the healthy elderly and those with dementia. It is sensitive to levels of dementia and identifies changes over time (Bar-Haim Erez \& Katz, 2004). Additionally, moderate to high and statistically significant correlations $(r=0.38-0.55)$ were found between the LOTCA-G areas and the Mini Mental Status Examination (Folstein \& Folstein, 1975) (MMSE) total score (Bar-Haim Erez \& Katz, 2004). Once the authors granted their permission, the LOTCA-G was translated into Spanish. Two native speakers trained in OT translated the scale from English to Spanish and Spanish to English to ensure that the meaning was kept. Internal consistency reliability was tested for the final Spanish version and showed a Cronbach's alpha coefficient of 0.89 , which exceeded the recommended value of 0.70 based on previously published criteria (Nunnally, 1978). 
Table 3. Cognitive functions along the four assessment times of the study $(n=21)$

\begin{tabular}{|c|c|c|c|c|c|c|c|c|}
\hline & \multicolumn{2}{|l|}{$\mathrm{T}_{1}$} & \multicolumn{2}{|l|}{$\mathrm{T}_{2}$} & \multicolumn{2}{|l|}{$\mathrm{T}_{3}$} & \multicolumn{2}{|l|}{$\mathrm{T}_{4}$} \\
\hline & Median (Q1Q3) & Mean ranka & Median (Q1Q3) & Mean ranka & Median (Q1Q3) & Mean ranka & Median (Q1Q3) & Mean ranka \\
\hline \multicolumn{9}{|l|}{ Orientation } \\
\hline Place & $6(6-7)$ & $2.26^{*}$ & $6(6-7)$ & $2.74 *$ & $6(6-7)$ & $2.26^{*}$ & $6(6-7)$ & $2.74 *$ \\
\hline Time & $5(4.5-6)$ & $1.69 * *$ & $6(5.5-6)$ & $3.07 * *$ & $5(5-6)$ & $2.02 * *$ & $6(5-6.5)$ & $3.21 * *$ \\
\hline \multicolumn{9}{|l|}{ Visual perception } \\
\hline Object identification & $4(4-4)$ & 2.50 & $4(4-4)$ & 2.50 & $4(4-4)$ & 2.50 & $4(4-4)$ & 2.50 \\
\hline Shape identification & $4(3.5-4)$ & 2.50 & $4(3.5-4)$ & 2.50 & $4(3.5-4)$ & 2.50 & $4(3.5-4)$ & 2.50 \\
\hline Overlapping figures & $3(3-3)$ & 2.48 & $3(3-3)$ & 2.57 & $3(3-3)$ & 2.38 & $3(3-3)$ & 2.57 \\
\hline Object constancy & $4(4-4)$ & 2.52 & $4(4-4)$ & 2.52 & $4(3.5-4)$ & 2.43 & $4(4-4)$ & 2.52 \\
\hline \multicolumn{9}{|l|}{ Spatial perception } \\
\hline On self & $3(3-3.5)$ & 2.45 & $3(3-4)$ & 2.55 & $3(3-3.5)$ & 2.45 & $3(3-4)$ & 2.55 \\
\hline On examiner & $2(2-3)$ & 2.33 & $2(2-3)$ & 2.62 & $2(2-3)$ & 2.43 & $2(2-3)$ & 2.62 \\
\hline Self and surrounding & $3(3-3)$ & 2.48 & $3(3-3)$ & 2.48 & $3(3-3)$ & 2.48 & $3(3-3)$ & 2.57 \\
\hline \multicolumn{9}{|l|}{ Motor praxis } \\
\hline Motor imitation 3 (3-3) & 2.45 & $3(3-3)$ & 2.55 & $3(2.5-3)$ & 2.36 & $3(3-3)$ & 2.64 & \\
\hline Utilization of objects 4 (3.5-4) & 2.45 & $4(3.5-4)$ & 2.55 & $4(3-4)$ & 2.45 & $4(3.5-4)$ & 2.55 & \\
\hline Symbolic actions $4(4-4)$ & 2.50 & $4(4-4)$ & 2.50 & $4(4-4)$ & 2.50 & $4(4-4)$ & 2.50 & \\
\hline \multicolumn{9}{|l|}{ Visuomotor } \\
\hline Copy geometric forms & $4(3.5-4)$ & 2.50 & $4(3.5-4)$ & 2.50 & $4(3.5-4)$ & 2.50 & $4(3.5-4)$ & 2.50 \\
\hline Twodimension model & $3(2-3)$ & 2.50 & $3(2-3)$ & 2.50 & $2(2-3)$ & 2.40 & $3(2-3)$ & 2.60 \\
\hline Pegboard construction & $3(3-3)$ & 2.40 & $3(3-3)$ & 2.50 & $3(3-3)$ & 2.50 & $3(3-3)$ & 2.60 \\
\hline Block design & $3(3-3)$ & 2.45 & $3(3-3)$ & 2.55 & $3(3-3)$ & 2.45 & $3(3-3)$ & 2.55 \\
\hline Puzzle reproduction & $2(2-2.5)$ & $2.29 *$ & $2(2-3)$ & $2.67 *$ & $2(2-3)$ & $2.38^{*}$ & $2(2-3)$ & $2.67 *$ \\
\hline Drawing a clock & $2(2-3)$ & 2.33 & $2(2-3)$ & 2.52 & $3(2-3)$ & 2.43 & $3(2-3)$ & 2.71 \\
\hline \multicolumn{9}{|l|}{ Thinking operation } \\
\hline Categorisation & $3(2-3)$ & 2.43 & $3(2-3)$ & 2.52 & $3(2-3)$ & 2.52 & $3(2-3)$ & 2.52 \\
\hline Pictorial sequencing & $3(2.5-3)$ & 2.45 & $3(2.5-3)$ & 2.55 & $3(2.5-3)$ & 2.45 & $3(2.5-3)$ & 2.55 \\
\hline \multicolumn{9}{|l|}{ Memory } \\
\hline A famous personality & $3(3-3)$ & 2.45 & $3(3-3)$ & 2.55 & $3(3-3)$ & 2.45 & $3(3-3)$ & 2.55 \\
\hline A personal possession & $4(3-4)$ & 2.50 & $4(3-4)$ & 2.50 & $4(3-4)$ & 2.50 & $4(3-4)$ & 2.50 \\
\hline Everyday objects & $3(2-3)$ & 2.36 & $3(2-3)$ & 2.55 & $3(2-3)$ & 2.45 & $3(2-3)$ & 2.64 \\
\hline Attention/concentration & $3(3-4)$ & $2.33^{*}$ & $4(3-4)$ & $2.81^{*}$ & $3(3-4)$ & $2.14^{*}$ & $4(3-4)$ & $2.71^{*}$ \\
\hline
\end{tabular}

Notes. Values based on the Loewenstein Occupational Therapy Cognitive Assessment-Geriatric (LOTCA-G).

$\mathrm{T}_{1}$ : before the intervention; $\mathrm{T}_{2}$ : after completion of the first intervention period; $\mathrm{T}_{3}$ : after the intervention withdrawal period; $\mathrm{T}_{4}$ : after completion of the second intervention period; $\mathrm{Q} 1$ : first quartile; $\mathrm{Q} 3$ : third quartile. Bold values denote statistical significance: ${ }^{*} p \leq 0.05 ;{ }^{* *} p<0.001$

Friedman's test. 


\subsection{Intervention and data collection}

The home-based OT intervention followed a holistic, biopsychosocial and client-centred approach. According to the holistic view, it focused on the person as a unique and complex being who is shaped by her or his life history, habits, values and desires, also highlighting the importance of considering the sociocultural contexts relevant to each individual (Finlay, 2001). The primary aim of the intervention was to optimise the daily functioning of the subject with AD. The World Health Organization (2001) has postulated that functioning in daily life is the result of a bidirectional and dynamic interaction between the person and the context; in line with this biopsychosocial model, our OT strategy addressed the physical, psychological, social and environmental factors that influence functioning. Moreover, the intervention followed the client-centred process described by the American Occupational Therapy Association (2014) and by Schaber and Lieberman (2010), characterised by the active participation of the client-caregiver dyad in the intervention and by placing the individual needs of the participant with $\mathrm{AD}$ at the core of the OT process. The participants identified what occupations and tasks were meaningful to them, as well as their interests, problems and priorities regarding daily functioning. In discussion with the occupational therapist, they established what activities they wanted to train or maintain, and the intervention targeted those occupations that were important to the individual.

On the basis of the specific needs concerning each subject, the intervention aimed to enhance or maintain the greatest possible independence in ADL and optimise participation in meaningful occupations, through a multicomponent approach including activities and techniques in the following domains (see Supporting Information Table S1): (a) meaningful activities/tasks, (b) cognitive stimulation (e.g. reminiscence and reality orientation), (c) activation of psychomotor and sensory skills, (d) home modification, (e) caregiver counselling, and (f) training in daily living skills. All participants received an individualised combination of all six types of intervention domains, adapted on a case-by-case basis. OT was a twice weekly intervention, so it was an intensive program. All sessions were delivered in the participant's own home to a dyad including the person with $\mathrm{AD}$ and her/his caregiver, lasting about 90 min each session: (a) 5 min of space/time orientation; (b) about $30 \mathrm{~min}$ of activities/tasks directed towards the main objective of each session, established according to the specific needs of each participant; (c) about $30 \mathrm{~min}$ for ADL training; (d) $10 \mathrm{~min}$ to relax; and (e) $15 \mathrm{~min}$ of caregiver counselling. The intervention was conducted by a single trained occupational therapist with extensive experience of working with people with AD.

The study was carried out over a period of 6.5 months: 12 weeks for the OT intervention, 6 weeks for the treatment withdrawal and, subsequently, 8 weeks for the replication of the intervention. Before the beginning of the intervention, an initial assessment $\left(T_{1}\right)$ of the patterns of daily living, life experiences, preserved skills, environments, individual needs and priorities was conducted during two 1-hr sessions. All participants were evaluated on four occasions: initial assessment $\left(\mathrm{T}_{1}\right)$, before the treatment withdrawal period $\left(\mathrm{T}_{2}\right)$, when this withdrawal period was over $\left(\mathrm{T}_{3}\right)$ and immediately after replication of the intervention $\left(\mathrm{T}_{4}\right)$. The BI was applied at the $\mathrm{T}_{1}$ and $\mathrm{T}_{4}$ assessments. The LOTCA-G was applied in all four evaluations. Additionally, the participants with $\mathrm{AD}$ and their caregivers were asked to rate their degree of satisfaction with the OT program on a scale from 1 (very dissatisfied) to 10 (very satisfied) at $\mathrm{T}_{4}$. Assessments were carried out by one of the authors (AA).

\subsection{Data analysis}

Descriptive statistics were used to summarise the findings. The Kolmogorov-Smirnov test was used to determine the normal distribution. Age followed a normal distribution, so it was described using the mean and the standard deviation (SD). Categorical variables were reported as frequencies and percentages. Ordinal variables were described using median and the first and third quartiles (Q1-Q3).

The changes in the scores on the BI between $\mathrm{T}_{1}$ and $\mathrm{T}_{4}$ were tested for significance by means of the Wilcoxon's signed-rank nonparametric test. Friedman's nonparametric analyses were conducted to test for differences in the LOTCA-G subtests from $\mathrm{T}_{1}$ to $\mathrm{T}_{4}$, and the Wilcoxon's test was used as post hoc procedure to evaluate the changes after each of the two intervention periods $\left(T_{1} v s . T_{2}\right.$ and $\left.T_{3} v s . T_{4}\right)$. Bonferroni corrections for multiple comparisons $(0.05 / 2)$ were applied to prevent type I errors. The effect size $(r)$ [ES $(r)]$ of the changes in scores on the BI and the LOTCA-G subtests calculated by dividing the $Z$ of the Wilcoxon's tests by the square root of the total number of observations. An $\operatorname{ES}(r)$ of 0.10 constitutes a small effect, 0.30 medium effect and 0.50 large effect (Cohen, 1988). For all tests except the Wilcoxon post hoc test, the level of significance was set a priori at $p \leq 0.05$ (two-sided). The IBM SPSS 22.0 was used for the statistical analysis. 


\section{RESULTS}

All participants completed the whole intervention programme. The socio-demographic characteristics of the 21 participants with $\mathrm{AD}$ are shown in Table 1 . The mean age was 78.6 years $(\mathrm{SD}=3.3)$. Most participants were married women of secondary educational level and lived with others at home, in an urban area.

\subsection{Satisfaction with the intervention}

After completing the home-based OT intervention, on a scale from 1 to 10 , the degree of satisfaction with the program was the maximum (median) in the participants with $\mathrm{AD}$ (range 8-10, Q1-Q3 = 9-10), as well as in the sample of caregivers (range 9-10, Q1-Q3 = 10-10).

\subsection{Effects on functional independence}

There was a statistically significant change in the total score of the BI between the $T_{1}$ and $T_{4}$ assessments: 14 subjects improved their total score and 7 remained unchanged. The median score was 90 in the $\mathrm{T}_{1}$ (range 75-100, Q1-Q3 = 82.5-90) and $\mathrm{T}_{4}$ (range 80-100, Q1-Q3 = 85-95) assessments $(p<0.001)$, and the $\mathrm{ES}(r)$ was 0.55 . Regarding the ADL assessed by the BI, Table 2 shows changes in BI scores between the $\mathrm{T}_{1}$ and $\mathrm{T}_{4}$ assessments: the percentage of independent participants increased in the activities of dressing, bathing, grooming and toileting, with a statistically significant improvement of functional independence in dressing $[p=0.003 ; \operatorname{ES}(r)=0.46]$.

\subsection{Effects on cognitive functions}

Table 3 presents changes in the LOTCA-G during the study period. Friedman's analyses showed a statistically significant change in four subtests from $\mathrm{T}_{1}$ to $\mathrm{T}_{4}$ times: place orientation $(p=0.003)$, time orientation $(p<0.001)$, reproduction of a puzzle $(p=0.017)$ and attention/concentration $(p=0.011)$. Regarding these four LOTCA-G subtests, Table 4 details the level of significance and the ES( $r)$ of changes after each of the two intervention periods $\left(T_{1}\right.$ vs. $T_{2}$ and $T_{3}$ vs. $\left.T_{4}\right)$. The improvement in the temporal orientation reached statistical significance in the two comparisons made in this post hoc procedure and the effect was substantial. With respect to place orientation and attention/concentration functions, a statistically significant moderate improvement was identified in all comparisons. The change in the subtest of puzzle reproduction did not reach statistical significance. 
Table 4. Cognitive functions: changes after completion of the first and the second period of intervention $(n=21)$

\begin{tabular}{|c|c|c|c|c|}
\hline \multirow{2}{*}{ LOTCA-G subtest $^{\mathrm{a}}$} & \multicolumn{2}{|l|}{$T_{1}$ versus $T_{2}$} & \multicolumn{2}{|l|}{$T_{3}$ versus $T_{4}$} \\
\hline & $p$-value & $\mathrm{ES}(r)$ & $p$-value & $\mathrm{ES}(r)$ \\
\hline \multicolumn{5}{|l|}{ Orientation } \\
\hline Place & 0.025 & 0.34 & 0.025 & 0.34 \\
\hline Time & $p<0.001$ & 0.55 & $p<0.001$ & 0.56 \\
\hline \multicolumn{5}{|l|}{ Visuomotor } \\
\hline Puzzle reproduction & 0.046 & 0.31 & 0.083 & - \\
\hline Attention/concentration & 0.025 & 0.34 & 0.014 & 0.38 \\
\hline
\end{tabular}

Notes. Bold values denote statistical significance after Bonferroni correction $(p \leq 0.025)$.

$\mathrm{T}_{1}$ : before the intervention; $\mathrm{T}_{2}$ : after completion of the first intervention period; $\mathrm{T}_{3}:$ after the intervention withdrawal period; $\mathrm{T}_{4}$ : after completion of the second intervention period; $\operatorname{ES}(r)$ : effect size.

${ }^{a}$ The four subtests with statistically significant changes from $\mathrm{T}_{1}$ to $\mathrm{T}_{4}$ assessments.

\section{DISCUSSION}

The main contribution of this study was to determine the impact of a novel home-based OT intervention which is substantially different compared to the scarce literature on this topic. Our program was longer and more intense: in similar previous studies, the duration of the OT intervention ranged from 5 (Gitlin et al., 2001) to 18 (Callahan et al., 2017) home visits within a period of between 3 and 24 months respectively. Furthermore, this strategy was unique in terms of its content (biopsychosocial and meaningful occupation-based approach), the inclusion of an intervention withdrawal period and the selection of people at an early stage of the disease. While the previous literature on the home-based OT was characterised by the inclusion of heterogeneous samples, composed mainly of people with mild to moderate dementia, this study was the first to explore the effects of a home-based OT program in a sample made up only of people in a mild stage of dementia, according to the GDS staging. In support of our hypothesis, we found evidence that the OT program was effective in terms of performance in ADL and several cognitive functions.

Regarding self-care activities, the results showed a statistically significant improvement in the total value of the BI after the OT program. In similar samples of people with mild $\mathrm{AD}$, prospective studies have found a progressive decrease in the ability to perform ADL over a period of time ranging from 6 months (Arrighi, Gélinas, McLaughlin, Buchanan, \& Gauthier, 2013; Cortes et al., 2008; Wattmo, Minthon, \& Wallin, 2016) to 1 year (Hallikainen et al., 2013). For example, in a sample with a MMSE initial mean score of 20 points, the participants decreased their independence in ADL significantly at 6 months (Cortes et al., 2008). In the current study, however, 6.5 months after the moment of inclusion, the participants significantly enhanced their functional skills. Moreover, despite the progressive nature of the $\mathrm{AD}$, the proportion of independent participants increased or remained in all the self-care activities studied at the final assessment. However, it should be noted that the results were only significant in the dressing activity. Two reasons may explain these findings. First, the main dysfunction in ADL was identified in dressing. Similarly, previous research has found that this activity deteriorates in a mild phase of dementia, while other daily activities such as transfers and feeding are intact in most people in the early stages (Cortes et al., 2008; Delva et al., 2014; Giebel et al., 2014; Giebel, Sutcliffe, \& Challis, 2015). The significant improvement identified in this activity was consistent with the relevance of the dressing disability within the limitations in self-care in the study population. Second, it is necessary to consider the good functional status of the participants at the beginning of the study. The initial median on the BI was 90 points and most previous studies have established this value as the cut-off point for an optimal 
functional ability (Diez-Ruiz et al., 2016; Granger, Dewis, Peters, Sherwood, \& Barrett, 1979; Patel, Duncan, Lai, \& Studenski, 2000; Uyttenboogaart, Stewart, Vroomen, De Keyser, \& Gert-Jan Luijckx, 2005). In addition, a recent study among community-dwelling older people established that independent living in the home is possible with a total score $\geq 85$ points (Schulc, Pallauf, Mueller, Wildbahner, \& Them, 2015). Therefore, the sample was already functioning at a high level of independence at initial assessment. Most participants were independent in eight of the ten activities analysed, which showed that the potential for improvement in ADL was limited.

The positive impact on functional independence was in line with the improvement in ADL found in a sample of people with mild to moderate dementia after receiving a community-based OT program (Graff et al., 2006). However, unlike our study, two RCT did not find a significant improvement in ADL after a home-based OT intervention (Callahan et al., 2017; Gitlin et al., 2001). Two possible explanations are the low intensity of the interventions implemented in these studies and the inclusion of people with a high level of dependence. In the work of Callahan et al. (2017), the participants had significant impairments in daily activities at baseline and received a total of 18 in-home sessions over a very long period ( 2 years). The program evaluated by Gitlin et al. (2001) involved only five home visits and one of the inclusion criteria was to report dependence of the person with dementia in at least two ADL; in addition, unlike our study, the intervention was only provided to the caregivers, without including training activities for the person with dementia, and it is difficult, therefore, to compare our results with those obtained in this controlled trial. On the other hand, with regard to day care services for people with dementia, research on the impact of OT programs has not found significant improvements in ADL (Baldelli et al., 2007; Cho et al., 2015; Lam et al., 2010; Ng et al., 2006). In contrast to the present study, these day care programs did not include home-based OT sessions and their structure was group-based. Therefore, instead of implementing the interventions in a clinical setting, our positive results suggest the importance of training the performance of daily activities in the real and usual environment of each person, with the utensils and objects that the participant uses in his/her daily life, a client-centred approach and involving the primary caregivers.

To our knowledge this study was the first specifically designed to measure the impact of a homebased OT program on the cognitive functions of people with AD. Moreover, the majority of OT research with this population has been performed using screening instruments such as the MMSE. However, the LOTCA-G was used because it allows the obtaining of a more in-depth cognitive profile, with appropriate psychometric properties to measure changes after the intervention (Bar-Haim Erez \& Katz, 2004). The participants showed significant moderate-to-substantial improvements in orientation (place and time) and attention/concentration skills after each of the two intervention periods. No statistically significant changes were found in other cognitive functions over time although the analyses revealed that all these variables tended to improve or maintain the scores of the first assessment when the program was implemented. Our results were consistent with those of previous studies examining OT strategies in other settings such as a geriatric long-term therapy unit (Bach et al., 1995) or day care centres (Cho et al., 2015; Lim et al., 2012; Ng et al., 2006). However, Baldelli et al. (2007) did not find improvements in cognition after an OT program in a day care centre; the inclusion of people with moderately severe dementia and the differences in the mean age of the samples may contribute to the dissimilarity between the two studies ( 85 years vs. 78 in our study). Finally, the intervention withdrawal period was applied to check whether the effects of the treatment in the cognitive area remained. However, the improvements were not sustained after the program stopped. In most cognitive subtests, values decreased from $T_{2}$ to $T_{3}$, between the beginning and the end of the intervention withdrawal period, and rose again in $\mathrm{T}_{4}$. Therefore, according to these results, a practical implication of the study could be the need to implement this OT intervention in a continuous way to optimise results and facilitate the maintenance of its effects on cognitive functioning. 


\subsection{Limitations}

Some limitations warrant further consideration. We must emphasie the lack of a control group. The improvements could have been achieved through the OT program, since the participants had a progressive disease and none of them received another type of nonpharmacological intervention. However, due to the uncontrolled design, the possible influence on the results of pharmacological treatment or other interventions such as primary healthcare consultations cannot be ruled out. Another concern is generalisability. The relatively small number of participants, female predominance and the fact that the population was selected using a nonrandom sampling technique limited the generalisability of the findings to all community-dwelling older adults with mild AD. However, regarding the sample size, a previous review on the appropriate size for pilot studies concluded that a sample of 20-25 participants is adequate if the objective is to evaluate the effectiveness of the intervention in a single group (Hertzog, 2008). The sample was recruited from hospital outpatients and participation in the study intervention was voluntary. This recruitment procedure may implicate a selection of a subgroup of people with AD, i.e. those who are more motivated to seek more comprehensive care and to receive new interventions. Furthermore, most participants lived in urban areas, thus making it difficult to generalise the results for people living in rural contexts. Lastly, given that the final assessment was done immediately after the OT program, it is not known whether the improvement shown in the sample lasted beyond the completion of the study.

\subsection{Implications for practice and future research}

The results of this study showed the benefits of initiating interventions in an early stage, as well as the importance of applying the OT programs in the home environment, in a more intensive way in comparison with previous studies, with a flexible protocol based on the interests and abilities of each person and a combined approach instead of focusing the intervention on solitary domains. Considering the seriousness of functional decline issues related to this health condition, even with the limitations of this study, our research suggests that more programs specifically like this should be run in the community for the following reasons. First, the intervention had a positive impact on daily activities and on some cognitive functions. The effect on functional independence in ADL was large. Therefore, this home-based OT program seems to promote autonomy in the self-care tasks, which allows participants to lead a more independent life in their own homes and, consequently, individuals may remain at home for a longer period of time. Furthermore, the acceptability of this intervention was noteworthy. The absence of dropouts among the sample after the start and the high levels of satisfaction perceived by the participants showed the optimal acceptance of the program. The comments of the occupational therapist who conducted the intervention supported this point, reporting that the prescribed activities appeared to be pleasurable and engaging for the vast majority of participants. We feel that some of the success of this program can be attributed to the fact that the intervention was based on the meaningful activities and specific needs of the target group, identified through a client-centred process. Lastly, this strategy may represent a cost effective use of scarce care resources for this growing population group, given the positive outcomes obtained, the small number of personnel involved in its implementation and the scarce use of supporting material. The greater proportion of expenditure was destined to the displacement of the professional to the participant's home, but this cost can be reduced including people residing in a more localised geographical area. Another strategy to facilitate the cost-effectiveness of the intervention could be the inclusion of periods in which the intervention is continued without the presence of the therapist in the home, promoting frequent contact between the therapist and the client-caregiver dyad through telephone conversations or other electronic information and communication technologies.

Further research is needed to confirm these preliminary findings. The expansion of this pilot study to a larger and broader sample in an experimental design with a control group for comparison would help to overcome the limitations mentioned above, providing a stronger evidence base for the effectiveness of this intervention protocol. The authors suggest the implementation of a RCT comparing this program to usual dementia care in Spain. In addition, the follow-up of participants over a time period is advantageous to determine the extent to which the intervention effects are maintained. Further research should also use 
qualitative research methods such as semistructured interviews with the primary caregivers to explore their perceptions about the experience of taking part in the study and their vision about the results of the intervention.

\section{CONCLUSIONS}

This research provided preliminary evidence for the effectiveness of an innovative home-based OT treatment in people with mild $\mathrm{AD}$ and its results give a great deal of information as a basis for further research. The findings demonstrated the positive effect of the intervention in improving functional independence in ADL. In addition, the participants showed improvements in several cognitive functions and a trend to maintain other cognitive skills. Given the irreversible and progressive nature of the AD and the relatively long duration of the total study period per participant, our research supported that this client-centred OT program may be an effective approach to delay functional disability and the loss of some cognitive functions, suggesting that a high-intensity home-based OT intervention can be beneficial as a nonpharmacological supplementary tool in health and social care for people with AD living in the community.

\section{CONFLICT OF INTEREST}

No conflicts of interest have been declared.

\section{REFERENCES}

Alzheimer's Association (2016). 2016 Alzheimer's disease facts and figures. Alzheimer's \& Dementia: the Journal of the Alzheimer's Association, 12, 459-509. https://doi.org/10.1016/j.jalz.2016.03.001

American Occupational Therapy Association (2014). Occupational therapy practice framework: Domain and process (3rd ed.). American Journal of Occupational Therapy, 68 (Suppl. 1), S1-S48. https://doi.org/10.5014/ajot.2014.682006

American Psychiatric Association (2000). Diagnostic and statistical manual of mental disorders (4th ed.). Washington, DC: American Psychiatric Association.

Arrighi, H. M., Gélinas, I., McLaughlin, T. P., Buchanan, J., \& Gauthier, S. (2013). Longitudinal changes in functional disability in Alzheimer's disease patients. International Psychogeriatrics, 25, 929-937. https://doi.org/10.1017/S1041610212002360

Bach, D., Bach, M., Bohmer, F., Fruhwald, T., \& Grilc, B. (1995). Reactivating occupational therapy: A method to improve cognitive performance in geriatric patients. Age and Ageing, 24, 222-226. https://doi.org/10.1093/ageing/24.3.222

Baldelli, M. V., Pradelli, J. M., Zucchi, P., Martini, B., Orsi, F., \& Fabbo, A. (2007). Occupational therapy and dementia: The experience of an Alzheimer special care unit. Archives of Gerontology and Geriatrics, 44, 49-54. https://doi.org/10.1016/j.archger.2007.01.007

Bar-Haim Erez, A., \& Katz, N. (2004). Cognitive profiles of individuals with dementia and healthy elderly: The Loewenstein Occupational Therapy Cognitive Assessment (LOTCA-G). Physical and Occupational Therapy in Geriatrics, 22, 29-42. https://doi.org/10.1080/J148v22n02_03

Callahan, C. M., Boustani, M. A., Schmid, A. A., LaMantia, M. A., Austrom, M. G., Miller, D. K., ... Hendrie, H. C. (2017). Targeting functional decline in alzheimer disease: A randomized trial. Annals of Internal Medicine, 166, 164-171. https://doi.org/10.7326/M16-0830

Cho, M. L., Kim, D. J., Chung, J. Y., Park, J. H., You, H. C., \& Yang, Y. A. (2015). Effects of a cognitiveenhancement group training program on daily living activities, cognition, and depression in the demented elderly. Journal of Physical Therapy Science, 27, 681-684. https://doi.org/10.1589/jpts.27.681

Cohen, J. (1988). Statistical power analysis for the behavioral sciences (2nd ed.). Hillsdale, NJ: Erlbaum.

Cortes, F., Nourhashémi, F., Guérin, O., Cantet, C., Gillette-Guyonnet, S., \& Andrieu, S., ...REAL-FR Group (2008). Prognosis of Alzheimer's disease today: A two-year prospective study in 686 patients from the REAL-FR Study. Alzheimer's and Dementia, 4, 22-29. https://doi.org/10.1016/j.jalz.2007.10.018 
Delva, F., Edjolo, A., Pérès, K., Berr, C., Barberger-Gateau, P., \& Dartigues, J. F. (2014). Hierarchical structure of the activities of daily living scale in dementia. The Journal of Nutrition, Health and Aging, 18, 698-704. https://doi.org/10.1007/s12603-014-0028-0

Diez-Ruiz, A., Bueno-Errandonea, A., Nuñez-Barrio, J., Sanchez-Martín, I., Vrotsou, K., \& Vergara, I (2016). Factors associated with frailty in primary care: A prospective cohort study. BMC Geriatrics, 16, 91. https://doi.org/10.1186/s12877-016-0263-9

Dooley, N. R., \& Hinojosa, J. (2004). Improving quality of life for persons with Alzheimer's disease and their family caregivers: Brief occupational therapy intervention. The American Journal of Occupational Therapy, 58, 561-569. https://doi.org/10.5014/ajot.58.5.561

Finlay, L. (2001). Holism in occupational therapy: Elusive fiction and ambivalent struggle. The American Journal of Occupational Therapy, 55, 268-276. https://doi.org/10.5014/ajot.55.3.268

Folstein, M. F., \& Folstein, S. E. (1975). Mini mental state: A practical method for grading the cognitive state of patients for clinician. Journal of Psychiatric Research, 12, 189-198. https://doi.org/10.1016/0022 3956(75)90026-6

Giebel, C. M., Sutcliffe, C., \& Challis, D. (2015). Activities of daily living and quality of life across different stages of dementia: A UK study. Aging and Mental Health, 19, 63-71. https://doi.org/10.1080/13607863.2014.915920

Giebel, C. M., Sutcliffe, C., Stolt, M., Karlsson, S., Renom-Guiteras, A., Soto, M., ... Challis, D. (2014). Deterioration of basic activities of daily living and their impact on quality of life across different cognitive stages of dementia: A European study. International Psychogeriatrics, 26, 1283-1293. https://doi.org/10.1017/S1041610214000775

Gitlin, L. N., Corcoran, M., Winter, L., Boyce, A., \& Hauck, W. W. (2001). A randomized, controlled trial of a home environmental intervention: Effect on efficacy and upset in caregivers and on daily function of persons with dementia. The Gerontologist, 41, 4-14. https://doi.org/10.1093/geront/41.1.4

Graff, M. J., Vernooij-Dassen, M. J., Thijssen, M., Dekker, J., Hoefnagels, W. H., \& Rikkert, M. G. (2006). Community based occupational therapy for patients with dementia and their care givers: Randomised controlled trial. British Medical Journal, 333, 1196-1199. https://doi.org/10.1136/bmj.39001.688843.BE

Granger, C. V., Dewis, L. S., Peters, N. C., Sherwood, C. C., \& Barrett, J. E. (1979). Stroke rehabilitation: Analysis of repeated Barthel Index measures. Archives of Physical Medicine and Rehabilitation, 60, 14 17.

Hallikainen, I., Hänninen, T., Fraunberg, M., Hongisto, K., Välimäki, T., Hiltunen, A., ... ALSOVA Study Group (2013). Progression of Alzheimer's disease during a three-year follow-up using the CERAD-NB total score: Kuopio ALSOVA study. International Psychogeriatrics, 25, 1335-1344. https://doi.org/10.1017/S1041610213000653

Hersen, M., \& Barlow, D. H. (1976). Single case experimental designs. New York, NY: Pergamon.

Hertzog, M. A. (2008). Considerations in determining sample size for pilot studies. Research in Nursing and Health, 31, 180-191. https://doi.org/10.1002/nur.20247

Itzkovich, M., Elazar, B., \& Katz, N. (1996). Geriatric version: Loewenstein occupational therapy cognitive assessment (LOTCA-G) battery. Pequannock, NJ: Maddak Inc.

Kazdin, A. E. (1980). Research design in clinical psychology. New York, NY: Harper and Row.

Kim, S. Y., Yoo, E. Y., Jung, M. Y., Park, S. H., \& Park, J. H. (2012). A systematic review of the effects of occupational therapy for persons with dementia: A meta-analysis of randomized controlled trials. NeuroRehabilitation, 31, 107-115. https://doi.org/10.3233/NRE-2012-0779

Korczak, D., Habermann, C., \& Braz, S. (2013). The effectiveness of occupational therapy for persons with moderate and severe dementia. GMS Health Technology Assessment, . https://doi.org/10.3205/hta000115

Lam, L., Lui, V., Luk, D., Chau, R., So, C., Poon, V., ... Ko, F. (2010). Effectiveness of an individualized functional training program on affective disturbances and functional skills in mild and moderate dementia-a randomized control trial. International Journal of Geriatric Psychiatry, 25, 133-141. https://doi.org/10.1002/gps.2309

Langa, K. M., Larson, E. B., Crimmins, E. M., Faul, J. D., Levine, D. A., Kabeto, M. U., \& Weir, D. R. (2017). A comparison of the prevalence of dementia in the United States in 2000 and 2012. JAMA Internal Medicine, 177, 51-58. https://doi.org/10.1001/jamainternmed.2016.6807

Lim, M. H. X., Liu, K. P. Y., Cheung, G. S. F., Kuo, M. C. C., Li, R., \& Tong, C. (2012). Effectiveness of a multifaceted cognitive training programme for people with mild cognitive impairment: a one-group preand posttest design. Hong Kong Journal of Occupational Therapy, 22, 3-8. https://doi.org/10.1016/j.hkjot.2012.04.002

Mahoney, F. I., \& Barthel, D. W. (1965). Functional evaluation: The Barthel Index. Maryland State Medical Journal, 14, 61-65.

McLaren, A. N., Lamantia, M. A., \& Callahan, C. M. (2013). Systematic review of non-pharmacologic interventions to delay functional decline in community-dwelling patients with dementia. Aging and Mental Health, 17, 655-666. https://doi.org/10.1080/13607863.2013.781121 
Ng, S., Lo, A., Lee, G., Lam, M., Yeong, E., Koo, M., ... Lau, V. (2006). Report of the outcomes of occupational therapy programmes for elderly persons with mild cognitive impairment (MCI) in community elderly centres. Hong Kong Journal of Occupational Therapy, 16, 16-22. https://doi.org/10.1016/S1569-1861(09)70035-X

Niu, H., Álvarez-Álvarez, I., Guillén-Grima, F., \& Aguinaga-Ontoso, I. (2016). Prevalence and incidence of Alzheimer's disease in Europe: A meta-analysis. Neurología (Barcelona, Spain), 32, 523-532. https://doi.org/10.1016/j.nrl.2016.02.016

Nunnally, J. C. (1978). Psychometric theory (2nd ed.). New York, NY: McGraw-Hill.

Patel, A. T., Duncan, P. W., Lai, S. M., \& Studenski, S. (2000). The relation between impairments and functional outcomes poststroke. Archives of Physical Medicine and Rehabilitation, 81, 1357-1363. https://doi.org/10.1053/apmr.2000.9397

Reed, C., Belger, M., Vellas, B., Andrews, J. S., Argimon, J. M., Bruno, G., ... Haro, J. M. (2016). Identifying factors of activities of daily living important for cost and caregiver outcomes in Alzheimer's disease. International Psychogeriatrics, 28, 247-259. https://doi.org/10.1017/S1041610215001349

Reisberg, B., Ferris, S. H., De Leon, M. J., \& Crook, T. (1982). The Global Deterioration Scale for assessment of primary degenerative dementia. The American Journal of Psychiatry, 139, 1136-1139. https://doi.org/10.1176/ajp.139.9.1136

Rogers, J. C., Holm, M. B., Burgio, L. D., Granieri, E., Hsu, C., Hardin, J. M., \& McDowell, B. J. (1999). Improving morning care routines of nursing home residents with dementia. Journal of the American Geriatrics Society, 47, 1049-1057. https://doi.org/10.1111/j.1532-5415.1999.tb05226.x

Schaber, P., \& Lieberman, D. (2010). Occupational therapy practice guidelines for adults with Alzheimer's disease and related disorders. Bethesda, MD: AOTA Press.

Schulc, E., Pallauf, M., Mueller, G., Wildbahner, T., \& Them, C. (2015). Is the Barthel Index an adequate assessment tool for identifying a risk group in elderly people living at home? International Journal of Nursing \& Clinical Practices, 2, 140. https://doi.org/10.15344/2394-4978/2015/140

United Nations Educational, Scientific and Cultural Organization (UNESCO) (2006). International standard classification of education: ISCED 1997. Montreal, QC: UNESCO- Institute for Statistics.

Uyttenboogaart, M., Stewart, R. E., Vroomen, P. C. A. J., De Keyser, J., \& Gert-Jan Luijckx, G. (2005). Optimizing cutoff scores for the Barthel Index and the modified Rankin scale for defining outcome in acute stroke trials. Stroke, 36, 1984-1987. https://doi.org/10.1161/01.STR.0000177872.87960.61

Wattmo, C., Minthon, L., \& Wallin, A. K. (2016). Mild versus moderate stages of Alzheimer's disease: Three-year outcomes in a routine clinical setting of cholinesterase inhibitor therapy. Alzheimer's Research and Therapy, 8, 7. https://doi.org/10.1186/s13195-016-0174-1

World Health Organization (2001). International classification of functioning, disability and health. Geneva, Switzerland: World Health Organization.

World Medical Association (2013). World Medical Association. Declaration of Helsinki. Ethical principles for medical research involving human subjects. The Journal of the American Medical Association, 310, 2191-2194. https://doi.org/10.1001/jama.2013.281053

Zhu, C. W., Leibman, C., McLaughlin, T., Zbrozek, A. S., Scarmeas, N., Albert, M., ... Stern, Y. (2008). Patient dependence and longitudinal changes in costs of care in Alzheimer's disease. Dementia and Geriatric Cognitive Disorders, 26, 416-423. https://doi.org/10.1159/000164797 